\title{
Ruptura de menisco associada à ruptura de ligamento cruzado cranial em cães
}

\author{
Association of meniscal lesion and cranial cruciate ligament insufficiency in dogs
}

\author{
Cássio Ricardo Auada FERRIGNO ${ }^{1}$; Daniela Fabiana Izquierdo CAQUIAS ${ }^{1}$; \\ Marcos Ishimoto Della NINA ${ }^{1}$; Olicies da CUNHA ${ }^{1}$; Kelly Cristiane ITO$^{1}$; \\ Tatiana Casimiro MARIANI ${ }^{1}$;Vanessa Couto de Magalhães FERRAZ ${ }^{1}$; Lourenço COTES ${ }^{1}$
}

${ }^{1}$ Departamento de Cirurgia da Faculdade de Medicina Veterinária e Zootecnia da Universidade de São Paulo, São Paulo- SP, Brasil

\begin{abstract}
Resumo
O trabalho descreve as lesões de menisco associadas com ruptura do ligamento cruzado cranial em 82 pacientes de diferentes raças, idades e pesos, com o objetivo de avaliar o tipo de ruptura de menisco associada a lesões do ligamento cruzado cranial. No período pré-operatório, os animais foram submetidos à avaliação clínica e radiológica. Todos os animais apresentaram ruptura total ou parcial do ligamento cruzado cranial; 21 (24,14\%) animais não apresentaram nenhuma lesão de menisco, os restantes (75,86\%) apresentaram apenas lesão no menisco medial. As lesões encontradas no menisco medial foram as seguintes: 33 joelhos (37,93\%) apresentaram eversão do corno caudal (Tipo 1); 15 (17,24\%) apresentaram lesão em alça de balde (Tipo 6); 3 (3,45\%) lesão de fibrilação (Tipo 4); 3 (3,45\%) ruptura longitudinal múltipla (Tipo 3); 3 (3,45\%) lesão longitudinal (Tipo 2); 1 (1,15\%) lesão tipo 7; e $10(11,49)$ apresentaram lesões múltiplas. A meniscectomia parcial do menisco medial foi realizada em $63(72,41 \%)$ joelhos e a meniscectomia total em $3(13,04 \%)$. O procedimento cirúrgico para a resolução da ruptura do ligamento cruzado cranial incluiu: avanço da tuberosidade tibial (TTA) (49 joelhos), osteotomia niveladora do platô tibial (TPLO) (15 joelhos), osteotomia em cunha da tíbia (CWO) (14 joelhos), extracapsular (quatro joelhos) e meniscectomia (cinco joelhos). Todos os casos evoluíram com o retorno à função habitual do membro pélvico acometido na primeira semana do período pós-operatório, e com a ausência de complicações. A alta porcentagem (75,86\%) de ruptura do menisco medial encontrada no presente trabalho demonstra a importância da avaliação prévia dos meniscos antes da realização da técnica de estabilização da articulação femorotibiopatelar.
\end{abstract}

Palavras-chave: Menisco. Ruptura do ligamento cruzado. Cão.

\begin{abstract}
The following study describes meniscus ruptures associated to cranial cruciate ruptures, in 34 dogs of different breeds, ages and weights. Before surgery the animals underwent clinical and radiographic examinations. All animals presented either total or partial cranial cruciate ruptures: 21 (24.14\%) of the animals didn't present any meniscus lesions, and the rest $(75.86 \%)$ presented only a medial meniscus lesion. The lesions found in the medial meniscus were the following: 33 stifles (37.93\%) presented with eversion of the caudal pole (Type 1), 15 (17.24\%) showed a bucked handle lesion (Type 6), $3(3.45 \%)$ presented with fibrillation lesion (Type 4), 3 (3.45\%) multiple fibrillation lesion (Type 3), 3 (3.45\%) longitudinal lesion (Type 2), 1 (1.15\%) lesion type 7 and 10 (11.49\%) presented multiple lesion. Surgical procedure for cranial cruciate rupture included: tibial tuberosity advancement (TTA) (49 stifle), tibial plateau leveling osteotomies (TPLO) (15 stifle), closing wedge osteotomy (CWO) (14 stifle), extracapsular (4 stifle) and meniscectomy alone (5 stifle), and all these techniques guaranteed weight baring and return to function in the first week after surgery, with no complications. Through this study we could demonstrate that meniscus tear is highly associated to cranial cruciate rupture and that the most common is type 1 (eversion of the caudal pole) and that cronicity of the lesion increases the probability of meniscus tear.
\end{abstract}

Keywords: Meniscus. Cranial cruciate ligament rupture. Dog.

Correspondência para:

Cássio Ricardo Auada Ferrigno

Departamento de Cirurgia da Faculdade de Medicina Veterinária e

Zootecnia (FMVZ) da Universidade de São Paulo (USP)

Av. Prof. Dr. Orlando Marques de Paiva, 87
São Paulo, SP, CEP 05508270

cassioaf@usp.br

Recebido: 27/06/2011

Aprovado: 16/08/2012 


\section{Introducão}

A articulação fêmoro-tíbio-patelar possui dois meniscos localizados lateralmente e medialmente no espaço articular femorotibial. Eles se apresentam em formato de "C" e são constituídos de fibrocartilagem. Encontram-se unidos à tíbia e ao fêmur por ligamentos: ligamento tibial cranial dos meniscos lateral e medial, ligamento tibial caudal dos meniscos lateral e medial, ligamento femoral do menisco lateral e ligamento intermeniscal. Além dos ligamentos, cada um dos meniscos está fixo, perifericamente, à cápsula articular, sendo que o menisco medial fixa-se ao ligamento colateral medial e possui menor mobilidade e maior porcentagem de lesões ${ }^{1}$. O terço externo de cada menisco tem aporte direto de sangue proveniente de vasos sanguíneos derivados da capsula articular, enquanto os outros dois terços internos são considerados avasculares ${ }^{2}$. Os meniscos têm a função de transmissão de cargas, absorção de energia, suplementação da estabilidade rotacional e varo-valga; lubrificação da articulação e manutenção da congruência das superfícies articulares ${ }^{3}$.

As rupturas de menisco em cães podem ocorrer de forma isolada, mas geralmente estão associadas à ruptura do ligamento cruzado cranial com o risco aumentando com a cronicidade da lesão $0^{4,5,6}$. O diagnóstico das lesões de menisco pode ocorrer quando o paciente deixa de sustentar o peso por duas semanas ou imediatamente após a ruptura de ligamento cruzado cranial. O proprietário pode ouvir ruído com som semelhante a "clic" ou crepitação quando o paciente salta ou anda, e este som pode ser audível durante as manobras de flexão e extensão no exame físico ${ }^{1}$. A ruptura é mais frequente em cães de raças grandes e gigantes; nas pequenas, a lesão é menos comum, possivelmente porque depois da ruptura do ligamento cruzado o membro locomotor é mantido elevado e em flexão, diminuindo as cargas sobre os meniscos $\cos ^{2,7,8,9}$. A maioria das rupturas de menisco é medial, envolvendo o corno caudal ${ }^{1,2,3,6}$. Em estudo realizado por Flo e De Young em 1978, observou-se incidência de lesões em 53\% dos casos de um total de 113 cães ${ }^{2}$.

Vários trabalhos têm associado a ruptura de menisco à ruptura do ligamento cruzado cranial, com classificação da lesão e cronicidade ${ }^{5,10,11,12,13,14,15}$. Bennett e May (1991) propuseram classificação da ruptura de menisco em sete tipos: Tipo 1 - desprendimento caudal com a eversão do corno caudal; Tipo 2 - ruptura longitudinal; Tipo 3 - ruptura longitudinal múltipla; Tipo 4 - fibrilação/degeneração da superfície; Tipo 5 ruptura axial; Tipo 6 - ruptura em alça de balde e Tipo 7 - ruptura transversal. Os tipos 1, 2 e 6 são os mais observados na clínica cirúrgica ${ }^{2,3,10}$.

A relação entre a ruptura de menisco associada à ruptura do ligamento cruzado cranial é conhecida em todo o mundo ${ }^{5,15}$, porém, não há estudos nacionais mencionando este contexto. Assim, o objetivo do estudo foi relatar a porcentagem de ruptura de menisco associada à ruptura de ligamento cruzado anterior encontrada na rotina no Laboratório de Ortopedia e Traumatologia Comparada do Departamento de Cirurgia da Faculdade de Medicina Veterinária e Zootecnia da Universidade de São Paulo, Brasil, e ressaltar a importância da sua avaliação mediante artrotomia minimamente invasiva no momento do procedimento cirúrgico.

\section{Material e Método}

O estudo foi prospectivo e incluiu 82 cães com diagnóstico de ruptura do ligamento cruzado cranial, avaliados no Laboratório de Ortopedia e Traumatologia Comparada do Departamento de Cirurgia da Faculdade de Medicina Veterinária e Zootecnia da Universidade de São Paulo, Brasil. No período pré-operatório, os animais foram submetidos à avaliação clínica mediante as manobras de gaveta e teste de compressão tibial. Uma vez diagnosticada a instabilidade articular 
por meio do exame físico, os animais foram submetidos à avaliação radiográfica do membro pélvico afetado. As radiografias foram realizadas em incidência latero-lateral, em duas posições, uma posicionando a articulação fêmoro-tíbio-patelar e tíbio-társica em ângulo de $90^{\circ}$; e a segunda, utilizando ângulo de $134^{\circ}$ na articulação fêmoro-tíbio-patelar e $90^{\circ}$ na articulação tíbio-társica. Estas posições radiográficas foram realizadas com o objetivo de se determinar o ângulo do platô tibial, a técnica cirúrgica mais adequada a ser empregada e o grau de osteoartrose. Todas as radiografias foram realizadas após a adequada sedação, utilizando acepromazina (Acepran ${ }^{\oplus}$, Vetnil/Univet, Louveira, São Paulo, Brasil), na dose de 0,05 mg/ kg e butorfanol (Torbugesic ${ }^{\varpi}$, Fort Dodge, Campinas, São Paulo, Brasil), 0,1 mg/kg, ambos pela via intramuscular. Para avaliar o estado geral dos pacientes, foram realizados previamente exames laboratoriais de hemograma completo, perfil renal e hepático.

A medicação pré-anestésica utilizada foi acepromazina $\left(0,05 \mathrm{mg} / \mathrm{kg}\right.$, via intramuscular) (Acepran ${ }^{\oplus}$, Vetnil/ Univet, Louveira, São Paulo, Brasil). A indução anestésica foi realizada com propofol (Propofol ${ }^{\oplus}$, Cristália, Itapira, São Paulo, Brasil) (5 mg/kg, via intravenosa) e a manutenção com isofluorano (Isofluorano ${ }^{\oplus}$, Abbott, Taboão da Serra, São Paulo, Brasil), sendo a fração aspirada do gás anestésico mantida entre 0,8 e 1,1 \%, adequando-se a porcentagem de acordo com o plano anestésico do animal. Todos os animais foram submetidos à anestesia epidural com lidocaína a 2\% (Xylestesin ${ }^{\oplus}$, Cistália, Itapira, São Paulo, Brasil) (5 mg/kg), associada à morfina (Morfina, Cristália, Itapira, São Paulo) $(0,1$ $\mathrm{mg} / \mathrm{kg}$ ) e fentanil (Fentanest ${ }^{\oplus}$, Cristália, Itapira, São Paulo, Brasil) (2 mcg/kg), sendo o volume final ajustado com solução fisiológica $(0,26 \mathrm{ml} / \mathrm{kg})$.

A região compreendida entre a pelve e a articulação tíbio-társica foi tricotomizada e realizada a antissepsia pré-cirúrgica. $\mathrm{O}$ animal foi posicionado em decúbito dorsal com o membro locomotor afetado sem restrição de movimento. Foi realizada incisão de pele parapatelar de aproximadamente $6 \mathrm{~cm}$ na região medial a patela. Posteriormente, a articulação foi abordada de forma minimamente invasiva com incisão de aproximadamente dois centímetros da cápsula articular, sem provocar a luxação da patela. Consecutivamente, expôs-se a articulação com afastador de Senn-Miller, a fim de posicionar a gordura infrapatelar de maneira adequada e obter melhor visibilização dos meniscos. Foram explorados tanto em sua parte cranial como caudal para a observação de possíveis rupturas ou alterações, assim como, confirmar a presença de ruptura do ligamento cruzado cranial total ou parcial. Nos pacientes em que existia lesão parcial de menisco, procedeu-se meniscectomia parcial, e no caso de ruptura total, a meniscectomia total foi realizada. Em todos os casos, a sutura da cápsula articular foi realizada em padrão de Mayo com fio de sutura monofilamentoso não absorvível (náilon $\mathrm{N}^{\circ} 0$ ). Após a sutura da cápsula articular e dos tecidos circunvizinhos, procedeu-se a realização da técnica cirúrgica de estabilização articular mais adequada ao paciente.

Após a sutura de rotina, o membro pélvico foi imobilizado com bandagem de conforto do tipo Robert Jones. Todos os animais foram avaliados com auxílio de radiografias no período pós-operatório imediato. A prescrição pós-operatória padrão constou de dipirona (Dipirona sódica ${ }^{\circledR} 500 \mathrm{mg}$, Laboratório Medley, São Paulo - SP, Brasil) (25mg/kg a cada 8 horas por 10 dias), carprofeno (Rimadyl ${ }^{\circledR} 25 \mathrm{mg}, 75 \mathrm{mg}$ ou $100 \mathrm{mg}$, Laboratório Pfizer, São Paulo - SP, Brasil) $(2,2 \mathrm{mg} / \mathrm{kg}$ a cada 12 por 10 dias), cloridrato de tramadol (Cloridrato de tramadol ${ }^{\circledR}$ 50mg, Laboratório Libbs Farm Ltda., São Paulo - SP, Brasil) (2mg/kg a cada 8 horas por 5 dias) e cefalexina (Cefalexina ${ }^{\oplus} 500 \mathrm{mg}$, Laboratório Medley, São Paulo - SP, Brasil) (30mg/kg a cada 12 horas por 10 dias). Os animais foram mantidos em repouso com restrição de espaço de 1,5 metro por 1,5 metro, por um período mínimo de 30 dias.

O primeiro controle pós-operatório foi realizado com sete dias, quando avaliou-se grau de inflamação, 
dor, funcionalidade e estabilidade articular. Para a avaliação da estabilidade articular, foi utilizado o teste de compressão tibial e gaveta. $\mathrm{O}$ segundo controle foi realizado com 14 dias de pós-operatório, sendo observados os mesmos parâmetros anteriores e remoção da sutura cutânea. No retorno de um mês pós-procedimento, além das avaliações regulares, foram realizados exames radiográficos, que no caso dos animais submetidos a procedimentos de osteotomia, se repetiram mensalmente até completar 90 dias do período pós-operatório, momento da alta médica.

\section{Resultados e Discussão}

Foram incluídos 82 animais neste estudo com diagnóstico de ruptura do ligamento cruzado cranial unilateral, dos quais cinco apresentaram ruptura do ligamento cruzado cranial bilateral, 28 machos e 54 fêmeas, totalizando 87 joelhos. As idades e pesos variaram de nove meses a 11 anos (média de 5,25 anos) e peso corporal de 4,7 a $68 \mathrm{~kg}$ (média de 30,9 kg), respectivamente. As raças incluídas foram: Labrador $(n=20)$, Rottweiler ( $n=11)$, Pit Bull $(n=8)$, Boxer $(n=7)$, Chow Chow $(n=6)$, Beagle $(n=3)$, Bulldog Inglês $(n=3)$, Dogo de Bordeaux $(n=2)$, Golden Retriver $(\mathrm{n}=2)$, Staffordshire Bull Terrier $(\mathrm{n}=1)$, Dogo Argentino $(n=1)$, Pastor Alemão $(n=1)$, Kuvasz $(n=1)$, Bernese $(n=1)$, Poodle $(n=1)$, Cane Corso $(n=1)$, Lulu da Pomerania $(n=1)$, Bichon Frisé $(n=1)$, Maltes $(n=1)$, Border Collie ( $n=1)$, e Akita $(n=1)$ e Sem Raça Definida $(n=8)$. Todos os animais apresentaram ruptura total ou parcial do ligamento cruzado cranial; 21 $(24,14 \%)$ animais não apresentaram nenhuma lesão de menisco, os restantes $(75,86 \%)$ apresentaram apenas lesão no menisco medial. As lesões encontradas no menisco medial foram as seguintes: 33 joelhos (37,93\%) apresentaram eversão do corno caudal (Tipo 1); 15 (17,24\%) apresentaram lesão em alça de balde (Tipo 6); três (3,45\%) lesão de fibrilação (Tipo 4); três $(3,45 \%)$ ruptura longitudinal múltipla (Tipo 3); três
(3,45\%) lesão longitudinal (Tipo 2); um (1,15\%) lesão tipo 7; e $10(11,49 \%)$ apresentaram lesões múltiplas. A meniscectomia parcial do menisco medial foi realizada em $63(72,41 \%)$ joelhos e a meniscectomia total em três (13,04\%).

Os procedimentos cirúrgicos para a resolução da ruptura ligamentar incluíram: osteotomia tibial em cunha (CWO) (14 joelhos), avanço da tuberosidade tibial (TTA) (49 joelhos), osteotomia niveladora do platô tibial (TPLO) (15 joelhos), meniscectomia (cinco joelhos), e extracapsular (quatro joelhos); conseguindo com todas as técnicas retorno a função normal do membro na primeira semana de pós-operatório, não havendo complicações concomitantes.

Um dos objetivos de nosso trabalho foi demonstrar a importância da avaliação minuciosa do menisco medial e lateral, visto que a não avaliação dos mesmos no momento da intervenção cirúrgica pode trazer intercorrências de claudicação aguda no período pós-operatório, tal como relatam Case et al. ${ }^{5}$. Há também relatos de claudicações a longo prazo. No presente trabalho, registrou-se alta porcentagem de ruptura de menisco $(75,86 \%)$ associada à ruptura do ligamento cruzado cranial. Portanto, as lesões de menisco apresentaram-se altamente relacionadas com a ruptura do ligamento cruzado cranial como relatam Ertelt e Fehr ${ }^{11}$ e Smith et al. ${ }^{14}$.

As lesões foram localizadas apenas no menisco medial. A lesão mais comum foi a eversão do corno caudal, coincidindo com os relatos de Necas e Zatloukal ${ }^{4}$, Austin et al. ${ }^{7}$ e Bennet e May ${ }^{10}$.

Uma vez que as lesões do menisco medial foram localizadas e visibilizadas, realizou-se a classificação pelo sistema de Bennet e May ${ }^{10}$. Esta classificação influi na decisão de meniscectomia total ou parcial. $\mathrm{O}$ tipo de meniscectomía está diretamente relacionado ao prognóstico, visto que, nas meniscectomias totais a osteoartrose tende a evoluir mais rapidamente em comparação com as meniscectomias parcias. 
O tratamento instituído para a ruptura parcial de menisco foi a meniscectomia parcial e para a ruptura total a meniscectomia total, corroborando o tratamento utilizado por Bennet e May ${ }^{10}$. A meniscectomia total deve ser evitada com a finalidade de um melhor prognóstico para o animal e para a funcionabilidade do joelho ${ }^{8}$.

A elevada porcentagem de recuperação pós-cirúrgica coincide com os resultados reportados por Case et al. ${ }^{5}$; a detecção precoce de ruptura de menisco e o tratamento cirúrgico evitam claudicações futuras após a estabilização articular.

Os resultados demonstram que a ruptura do ligamento cruzado cranial está associada à alta porcentagem de ruptura do menisco medial, sendo que a lesão mais frequente foi a eversão do corno caudal. Em futuros projetos, pretende-se correlacionar o tempo transcorrido desde a lesão do ligamento cruzado cranial até e ruptura do menisco medial. Estes dados permitirão prognósticos mais acurados, já que nas cirurgias onde preservam-se os meniscos, a evolução da osteoartrose é mais lenta e a biomecânica do joelho é preservada9

A alta porcentagem $(75,86 \%)$ de ruptura do menisco medial encontrada no presente trabalho de-

\section{Referências}

1. FLO, G. L. Meniscectomy. In: BOJRAB, M. J. (Ed.). Current techniques in small animal surgery. 3 ed. Philadelphia: Lea\&Febiger, 1990. p. 694-700.

2. DENNY, H. R.; BUTTERWORTH, S. J. Joelhos. In: DENNY, H. R. (Ed.). Cirurgia ortopédica em cães e gatos. 4 ed. São Paulo: Roca, 2000. p. 396-427.

3. SCHULZ, K. Diseases of the joint. In: FOSSUM, T. (Ed.). Small animal surgery. 3 ed. Louis: Mosby, 2007. p. 1285-1289.

4.NECAS, A.; ZATLOUKAL, J. Factors related to the risk of meniscal injury in dogs with cranial cruciate ligament rupture. Acta Veterinaria, v. 71, n. 1, p. 77-84, 2002.

5. CASE, J. B.; HULSE, D.; KERWIN, C.; PEYCKE, L. E. Meniscal injury following initial cranial cruciate ligament stabilization surgery in 26 dogs (29 stifles). Veterinary and Comparative Orthopaedics and Traumatology, v. 21, n. 4, p. 365-367, 2008.

6. VASSEUR, P. B. Stifle joint. In: SLATTER, D. (Ed.). Textbook of small animal surgery. 3. ed. Philadelphia: Sanders, 2003. p. 2095-2116.

7. AUSTIN, B.; MONTGOMERY, R. D.; WRIGHT, J.; BELLAH, J. R.; TONKS, C. Evaluation of three approaches to meniscal. Veterinary and Comparative Orthopaedics and Traumatology, v. 20, n. 2, p. 92-97, 2007. monstra a importância da avaliação prévia dos meniscos antes da realização da técnica de estabilização da articulação femoro-tíbio-patelar. A avaliação dos meniscos pode ser realizada no período pré-operatório por meio de exames de imagem como ultra-sonografia, tomografia computadorizada ou ressonância magnética. A ressonância magnética é efetiva na avaliação, porém, a acessibilidade ao exame é baixa ${ }^{16}$. No Brasil, a maioria das avaliações é possível por meio de artrotomia e, em menor escala, por artroscopia. Ressalta-se a importância de avaliar os meniscos em todos os tipos de intervenções cirúrgicas de correção de ruptura de ligamento, baseado na alta taxa de ruptura de menisco. O desconhecimento ou falta de avaliação de possível ruptura de menisco pode levar a período pós-operatório prolongado e sem melhoras na claudicação do membro pélvico afetado ${ }^{5}$.

Os resultados do presente estudo demonstraram a importância da artrotomia medial para exploração do menisco medial em cães com ruptura do ligamento cruzado cranial, devido ao alto índice de ruptura de menisco associada a esta doença.

8. POZZI, A.; TONKS, C. A.; LING, H. Y. Femorotibial contact mechanics and meniscal strain after serial meniscectomy. Veterinary Surgery, v. 39, n. 4, p. 482-488, 2010.

9. THIEMAN, K. M.; POZZI, A; LING, H. Y, LEWIS, D. Comparison of contact mechanics of three meniscal repair techniques and partial meniscectomy in cadaveric dog stifles. Veterinary Surgery, v. 39, n. 3, p. 355-362, 2010.

10. BENNETT, D.; MAY, C. Meniscal damage associated with cruciate disease in the dog. Journal of Small Animal Practice, v. 32, n. 3, p. 111-117, 1991.

11. ERTELT, J.; FEHR, M. Cranial cruciate ligament repair in dogs with and without meniscal lesions treated by different minimally invasive methods. Veterinary and Comparative Orthopaedics and Traumatology, v. 22, n. 1, p. 21-26, 2009.

12. GUASTELlA, D. B.; FOX, D. B.; COOK, J. L. Tibial plateau angle in four common canine breeds with cranial cruciate ligament rupture, and its relationship to meniscal tears. Veterinary and Comparative Orthopaedics and Traumatology, v. 21, n. 2, p. 125-128, 2008.

13. POZZI, A.; HILDRETH, B. E.; RAJALA-SCHULTZ, P. J. Comparison of Arthroscopy and Arthrotomy for Diagnosis of medial meniscal pathology: An ex vivo study. Veterinary Surgery, v. 37, n. 8, p. 749-755, 2008. 
14. SMITH, G. N.; MICKLER, E. A.; ALBRECHT, M. E.; MYERS, S. L.; BRANDT, K. D. Severity of medial meniscus damage in the canine knee after anterior cruciate ligament transaction. Osteoarthritis and Cartilage, v. 10, n. 4, p. 321$326,2002$.

15. HAYES, G. M.; LANGLEY-HOBBS, S. J.; JEFFERY, N. D. Risk factors for medial meniscal injury in association with cranial cruciate ligament rupture. Journal of Small Animal Practice, v. 51, n. 12, p. 630-634, 2010.

16. MARINO, D. J.; LOUGHIN, C. A. Diagnostic imaging of the canine stifle: A review. Veterinary Surgery, v. 39, n. 3, p. 284295, 2010. 